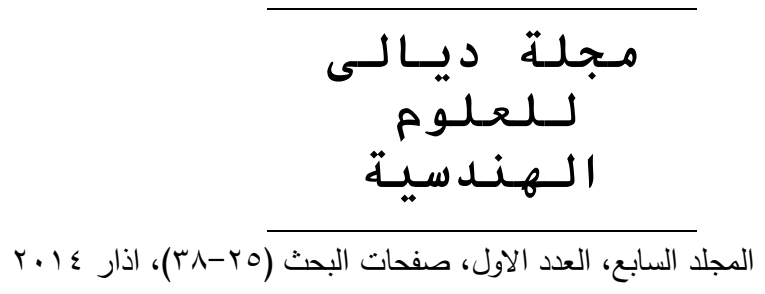

\title{
دراسة تحديد أطول فترة صلاحية لأسلاك تقويم الأسنان في أجواء مختلفة
}

\author{
محمد عبد اللطيف أحمد
}

مدرس مساعد/ هندسة الأنتاج والمعادن/ الجامعة التكنولوجية/ بغداد/ العراق

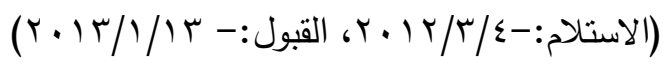

الخلاصة

يتلخص البحث بتحديد أطول فترة صـاحية لأسـالك تقويم الأسنان في أربع أجواء أكالة ، الأول جو مماثل لفم

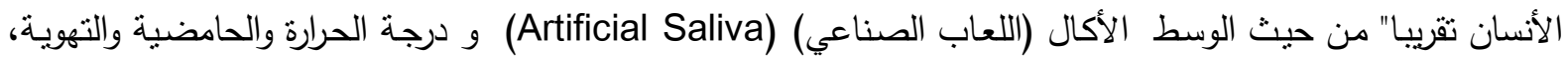

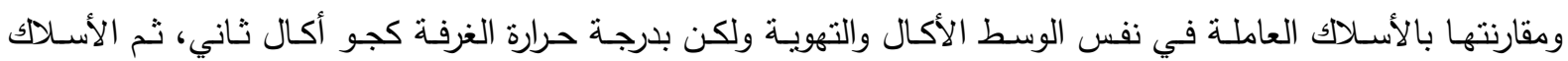
المتواجدة في محلول كلوريد الصوديوم (r,0\%wt NaCl) بدلا" عن اللعاب الصناعي وبدرجة حرارة الغرفة وبنفس التهوية كجو أكال ثالث وأخيرا" المقارنة مع الأسلاك المتواجدة في محلول كلوريد الصوديو الصوم وبدرجة حرارة جسم الأنسان ومعرضية

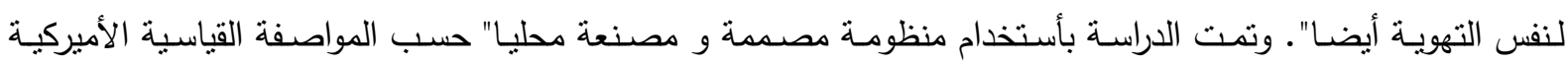

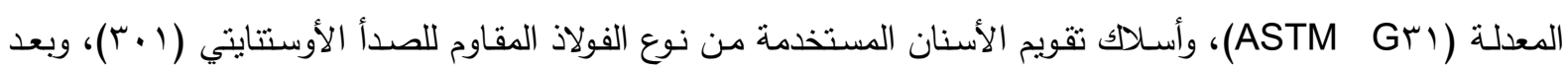
إجراء أختبارات التآكل الأربعة أعلاه و لفترة زمنية مقدارها ستة أثنهر منتالية وبأستخدام قانون معدل تغلغل أومعدل نفاذ تتقر التآكل ( Corrosion Penetration Rate) وبالطرق الرياضية التقليدية ومن الصور المجهرية للعينات قبل وبعد فئه

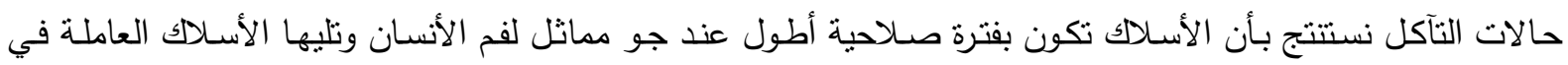

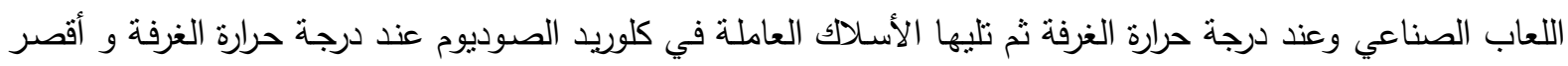

فترة صلاحية تكون للأسلاك العاملة في محلول كلوريد الصوديوم عند درجة حرارة جسم الأنسان. كلمات الدلالة : تقويم الأسنان، المواد البايولوجية، الفولاذ المقاوم للصدأ،الفولاذ الأوستتايتي، اللعاب الصناعي.

-

جميع المواد المعدنية المستخدمة كزوارع جراحية و تقويمية والتي تتضمن مواد ذات مقاومة جيدة للتآكل تعاني من أنحلال كيميائي أو ألكتروكيميائي وبمعدلات محدودة بسبب الوسط المعقد والأكال للجسم البشري ـ وسوائل الجسم تتألف من الماء ومركبات معقدة و أوكسجين مذاب وكمية كبيرة جدا"جدا" من كلوريد الصوديوم (NaCl) و محاليل أخرى تحوي وبمقدار كبير على أيونات الكلورايد (Cl) ومحاليل ألكتروليتية أخرى منل البيكاربونات و مقادير قلبلة من 
البوتاسيوم و الكالسيوم والمغنسيوم و الفوسفات والكبريت والأحماض الأمينية والبروتينات والبلازما والسوائل اللمفية

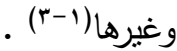

إن محلول كلوريد الصوديوم يعتبر من الأوساط المؤثرة على الفولاذ المقاوم للصدأ لأحتواءه على هالوجين أو هاليد (Clوالذي يعتبر من الأيونات الأساسية المولدة للتآكل التتقري ، ويستخدم محلول كلوريد الصوديوم في التجارب لكونه المحلول البسبط لدراسـة سلوك التآكل في الفولاذ المقاوم للصدأ وأضـافة لكونـه أحد مكونـات اللعاب ـ ومـن المعروف بـأن أيون

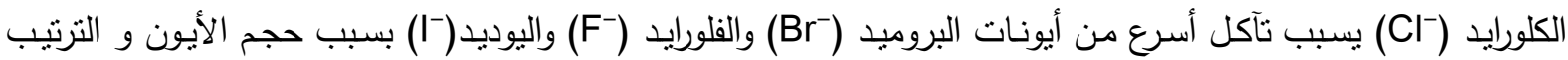
الأكتروني وحركة الأيونات و ألفتها الكيميائية ولهذا يعتبر أيون الكلورايد (Cl) مولدا" و محفزا" لأمكانية حصول التآكل التتقري و خصوصا" في الأوساط البطيئة الحركة أو الساكنة والمحتوية على الأوكسجين ومن الأمنلة الثائعة على ذلك هو التآكل الثديد للفولاذ المقاوم للصدأ المحتوي على عناصر سبك مثل الكروم (Cr) عند تعرضه لمحلول كلوريد الصوديوم والذي يولد كلوريد الكروم الذائب في المحلول الأكال وتاركا" نقاط لنضوب الكروم وهي نقاط فعالة وصغيرة الحجم مقارنة" بيقية السطح الكبير المحمي وبالتالي ستتولد النقر نتيجة زيادة سرعة التآكل في هذه النقاط الصغيرة (؛،ه). ومن المعروف إن أسلاك تقويم الأسنان المصنوعة من الفولاذ المقاوم للصدأ من أفضل الأسـلاك أستخداما". وبما إن للتأكل دور مهم في حياتتا اليومية وخصوصا" الصحية والطبية و إن الفولاذ المقاوم للصدأ ليس مقاوما" للصدأ في كل الظروف ؛ لذى ستكون هنالك علاقة بين التآكل وأسلاك نقويم الأسنان داخل فم الأنسان الحاوي على اللعاب (Saliva) والحاوي على نسبة ليست بقليلة من أيون الكلورايد (Cl- المحفز لتوليد التآكل التتقري في الفولاذ المقاوم للصدأ(1،ه). ولهذا سندرس حالات من التآكل في لعاب صناعي وفي محلول كلوريد الصوديوم عند درجة حرارة الجسم مرة وعند درجة حرارة الغرفة مرة أخرى وبوجود التهوية.

وتقويم الأسنان هو فرع تخصصي من علوم طب الأسنان الذي ينصب إهتمامسه على تطور الأسنان والفكين والوجه وطرق معالجة النتوهات في كل منها. هدف المعالجة التقويمية هو تحقيق صحة مثالية للفم عن طريق الحصول على إنطباق مثنالي للأسنان, و بالتنالي تحسين قدرة الأنسجة الفمويـة والسنية على مقاومـة الأمـراض والآفات وتحسين المظهر العام للوجه والإبتسامة مما يعزز تقة المريض بنفسة و بناءا" عليه يمكن تلخيص فوائد المعالجه التقويميه بتحسين الجماليات الوجهيـه بشكل عـام، والابتسـامة تحديدا"، تحسين العمل الوظيفي للأسنان و الأنسجة المحيطـة، منـع الأذى للأسنان الأمامية البارزة نتيجة للكدمات المفاجئة، معالجة الأسنان المطمورة والغير بازغة، وأخيرا" تحسين مقاومة الأسنان واللثة للآفات والأمراض(ب، (r).

وتقويم الأسنان معرف رسميا" مـن قبل الجمعيـة الأميركيـة لتقويم الأسنان بـأنها مجـال لطب الأسنان المعني بالإشراف والتوجيه والتصحيح لنمو الهياكل والمكونات العظمية للوجه والأسنان، بما في ذللك تلك الظروف التي تتطلب تحريك الأسنان أو تصحيح العلاقة بين الأسنان وعظام الوجه من تطبيق قوى معينة و (أو) تحفيز وتوجيه نمو عظام الوجه والأسنان ،لاحظ الشكل (1) ("). 


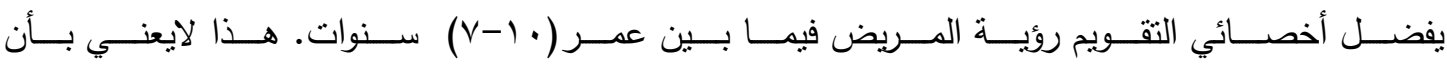

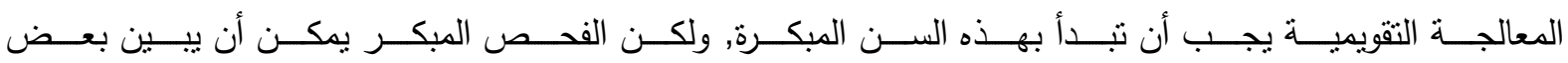

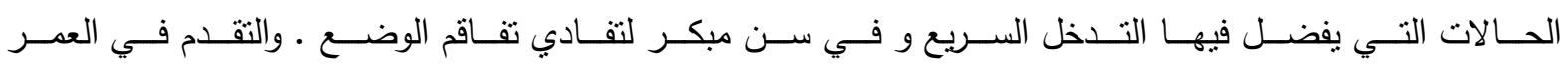

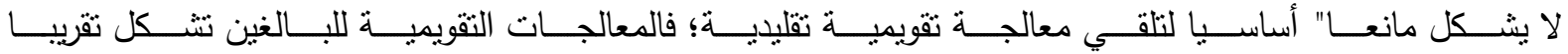

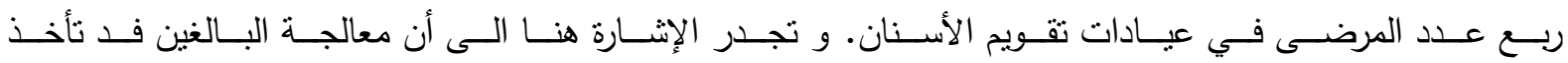

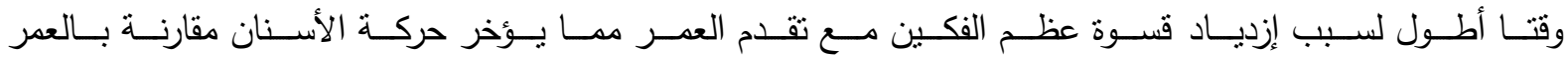
الأقل (0، (0).

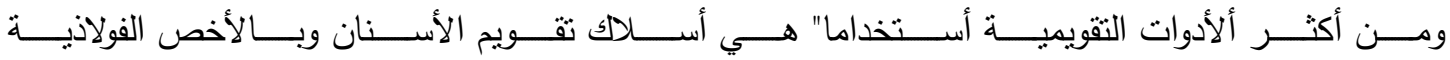

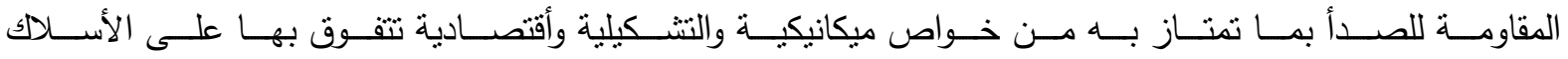

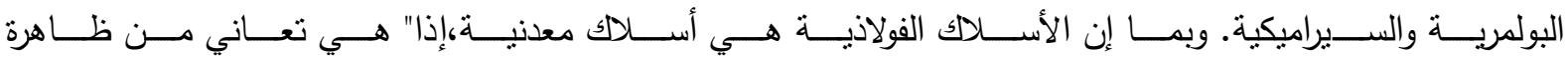

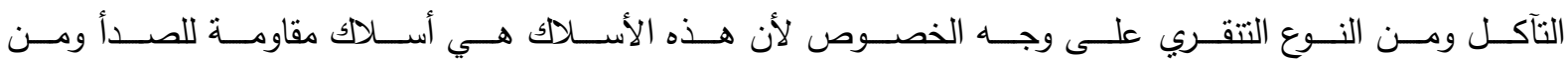

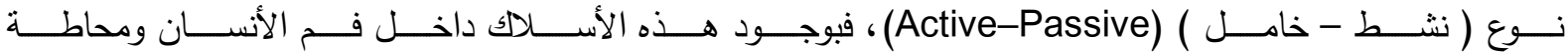

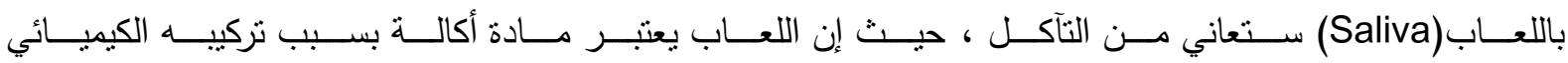

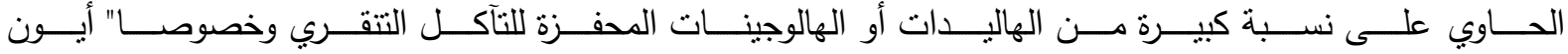

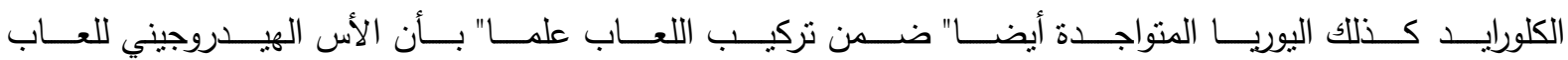

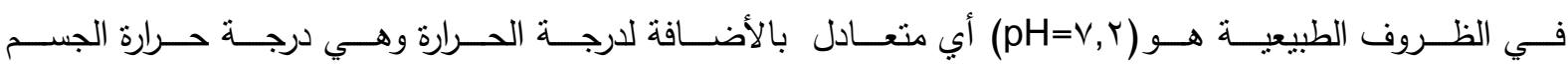

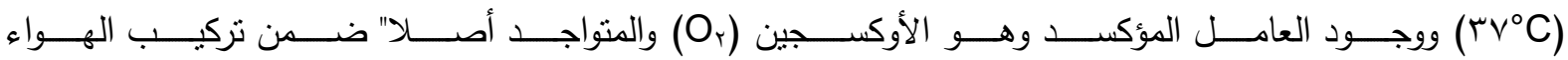

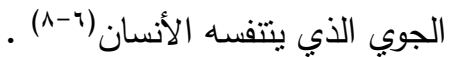

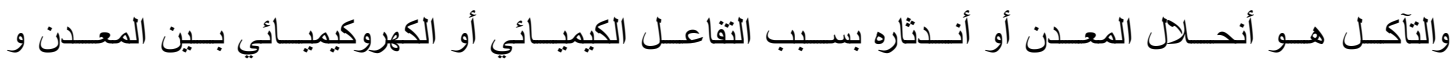

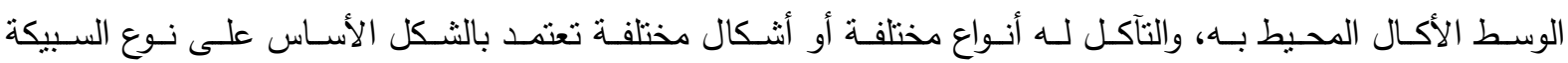

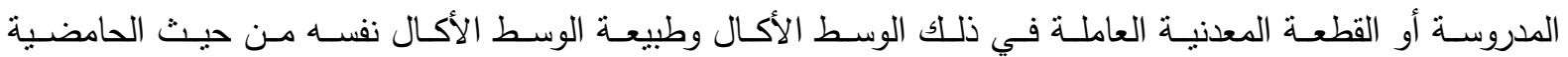

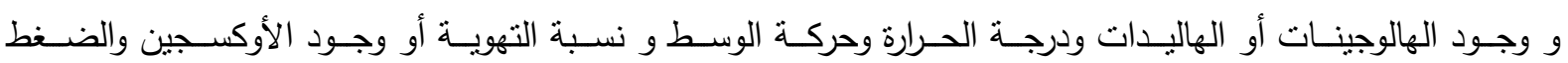
المحيط بالعملية وعوامل أخرى(-9 (l).

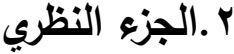

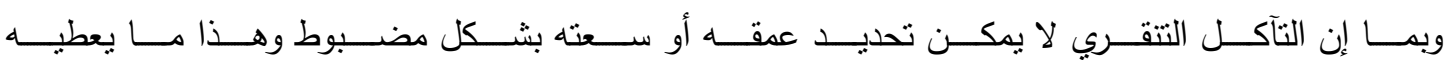

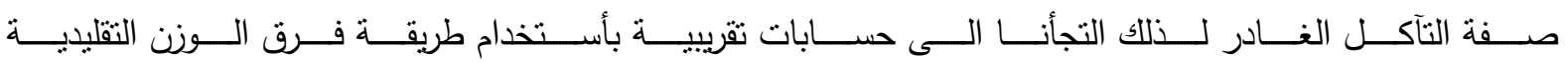
وبالصيغة الرياضية التالية (rا،r'):-

$$
\operatorname{CPR}(\mu \mathrm{m} / \mathrm{yr} .)=(\mathrm{KW} / \rho \mathrm{At})^{* 1 \cdot{ }^{r} \ldots \ldots \ldots . .(1)^{\left(1 r_{6} / r\right)}}
$$




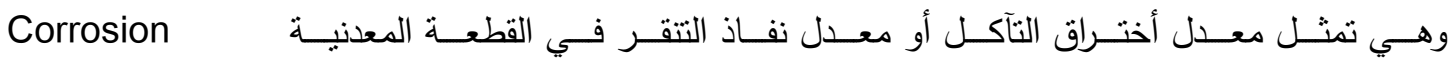
Penetration Rate(CPR) من K تابت مقداره NV,T وليس له وحدات. مقدار فرق الوزن بوحدة W

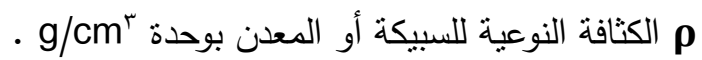
t فمن التعرض لوسط التآكل بوحدة hr

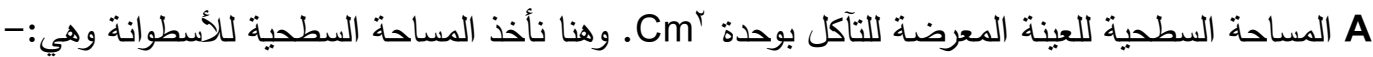
$A=r \pi(d / r) h$

حيث d هي قطر السلك و h طول السلك. ولمعرفة عمق التتقر التقريبي ( Penetration Depth) بعد فترة الأختبار والتي لا تتجاوز الستة أثشر نقوم

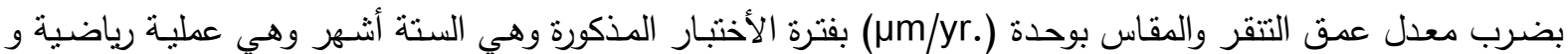
جبريـة نقليديـة ـ أي بعد تحديد معدل أختراق التآكل(CPR) نقوم بتخمين عمق التتقر أو عمق الأختراق(PD) رياضبا"

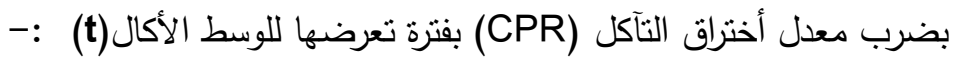

$\operatorname{PD}(\mu \mathrm{m})=\operatorname{CPR}(\mu \mathrm{m} / \mathrm{yr}). * \mathrm{t}(\mathrm{yr}$.

ولتخمين العمر التقريبي الكلي ( أو فتزة الصلاحية التقريبية ) (Piece Life ) لسلك تقويم الأسنان في أي وسط من أوساط الأختبار الأربعة يتم بالضرب الرياضي لمقلوب معدل عمق التتقر (PD) (P) بحاصل طرح عمق التتقر (PDR) من قطر السلك الكلي (d)، أي قطر السلك مطروحا" منـه عمق التتقر أو عمق الأختراق و مقسوم على معدل أختراق

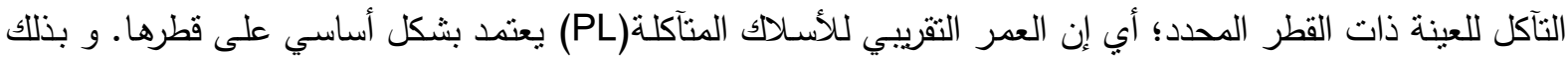
يصبح من اليسير تخمين فترة صـاحية أسلاكك تقويم الأسنان المدروسة خلال الفترة الزمنية المحددة و عن طريق العملية الرياضية التقليدية:-

$P L(y r)=.[d-P D(\mu m)] / C P R(\mu m / y r$.

ب.الجزء العملي

(, 1, 1, تحضير العينات

في هذا البحث سندرس التآكل التتقري الحاصل في أسـلاك تقويم الأسنان المصنوعة من الفولاذ الأوستنايتي المقاوم للصدأ( • • والتي نم الحصول عليها من مختبرات كلية طب الأسنان جامعة بغداد؛ وحسب المواصفة الأميركية

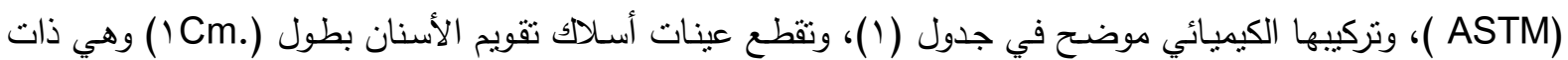
مقطع دائري و قطرها أصلا" ( l mm) ومن ثم أيجاد الوزن الأبتدائي لها وكذللك البنية المجهرية قبل حلات التآكل

يتم تهيئة و تحضير مادة اللعاب الصناعي (Artificial Saliva) والذي له نركيب كيميائي مبين في جدول (r) ويعتبر وسط أكال، وهو متعادل الحامضية (pH=v) وكذلك تهيئة المحلول الأكال الآخر وهو محلول كلوريد الصوديوم 


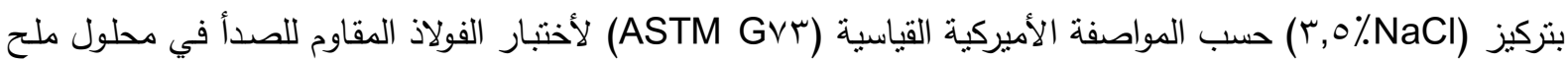

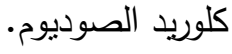

\section{r r r r تصنيع منظومة الأختبار}

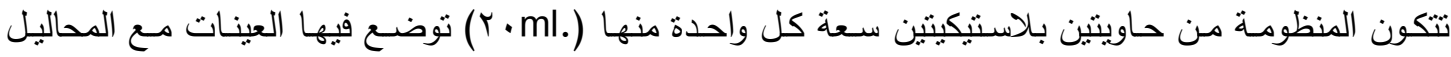

الأكالة (اللعاب الصناعي أومحلول كلوريد الصوديوم) والحاويتين موجودنين داخل حجرة الأختبار الزجاجية المقاومة للحرارة

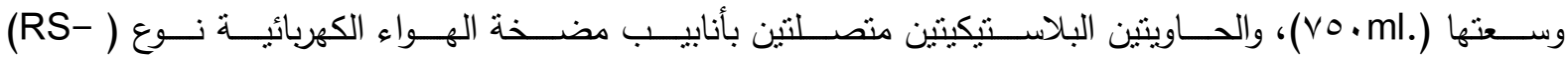

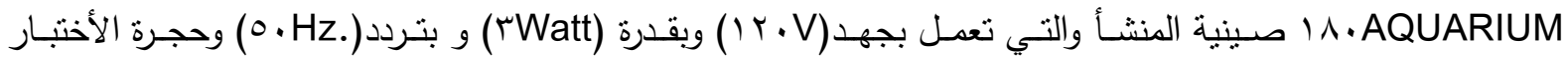

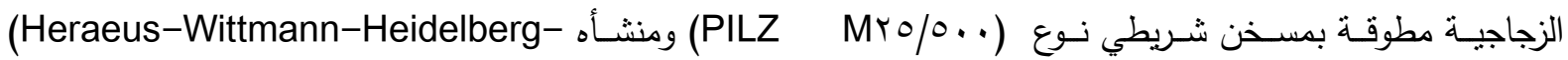

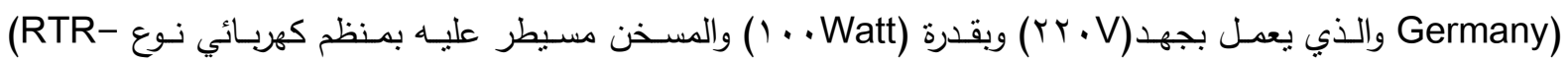

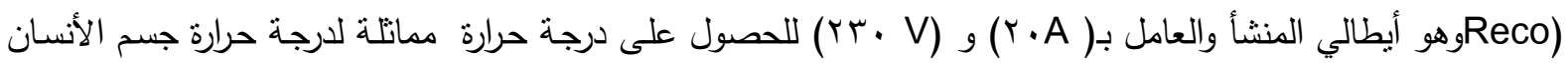

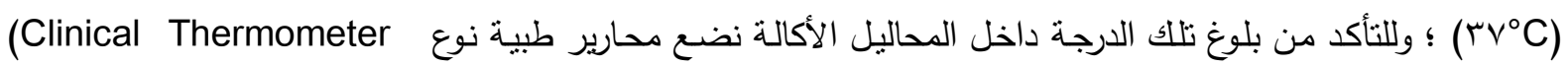
High Precision Pic Indolor - Artsana S.P.A Grandate(co)-ITALY)

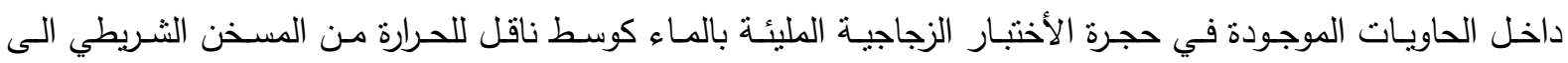

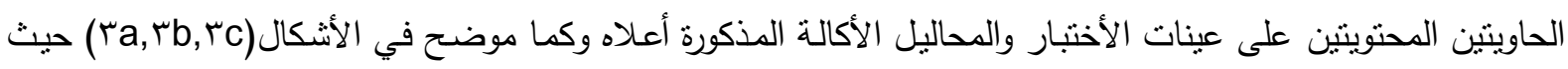

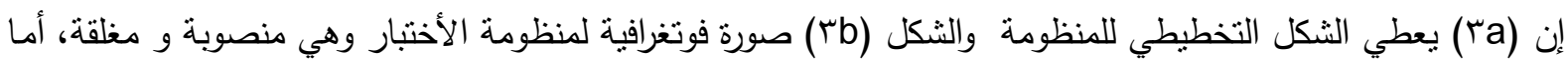

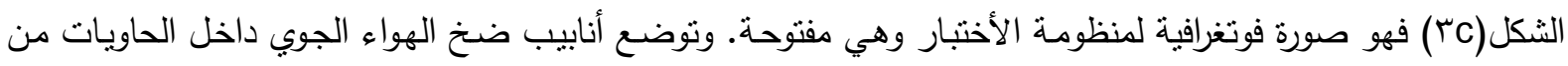
قاعدتها وذلك للحصول على نسبة كافية من غاز الأوكسجين rO المذاب داخل المحاليل الأكالة وبهذا يكون الظرف داخل هوهل

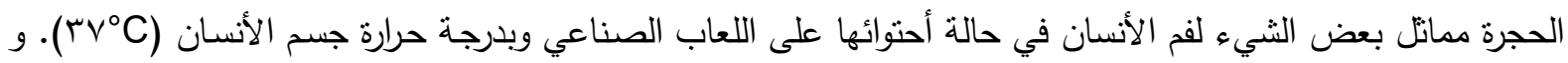

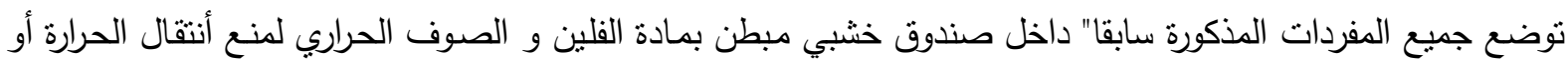

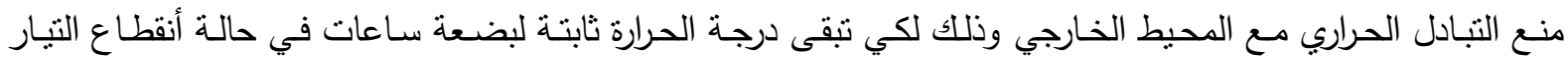
الكهربائي عن المسخن الحراري الشريطي المسبطر عليه و كذلك يتم ربط مضخة الهواء بوحدة مجهز القدرة الأضافية (Inputrr.V ac 0.Hz. \& Output rr.V ac 0.Hz.) ( Maxima Model Ir..VA) (UPS) للحيلولة دون أنقطاع تجهيز المنظومة بالأوكسجين لحين توفير مصدر كهربائي جديد.

؛ .أختبارات التآكل

بعد تحضير عينات من سلك تقويم الأسنان المذكور و أيجاد وزنها الأبتدائي، نقوم بغمر العينات في محاليل التآكل لحالات التآكل الأربعة الدذكورة, أبي بأربع مجاميع وفي كل مجموعة ثلاثنة عينات: المجموعة الأولى : العينات تكون في الجو المماثل لفم الأنسان والمتكون من اللعاب الصناعي (Artificial Saliva)

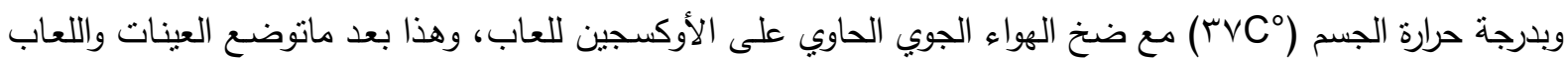
الصناعي في أحد حاويات منظومة الأختبار • الصنا

المجموعة الثانيـة: العينات في اللعاب الصناعي داخل حاوبـة أخرى غير تابعة للمنظومة أو خارجية ويضـخ لها الهواء الجوي بمضخة هواء كهربائية أخرى و بدرجة حرارة الغرفة.

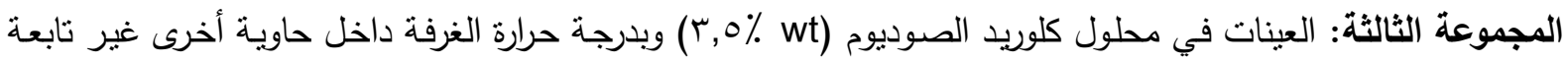
للمنظومة أو خارجية ويضخ لها الهواء الجوي بمضخة هواء كهربائية. 
المجموعة الرابعة: العينات في محلول كلوريد الصوديوم وبدرجة حرارة الجسم داخل أحدى حاويات المنظومة والتي تزود بالهواء الجوي.

وتبدأ التجربة بتوصيل منظومة الأختبار المذكورة بالمصدر الكهربائي لتبقى تحت المراقبة لمدة محددة ( ج أثهر في هذه التجربة)؛ ثم أخراج العينات بعد كل شهر و للأربع مجاميع ونجد فرق الوزن الحاصل بها ؛ أي تكرر عملية أيجاد فرق الوزن ستة مرات متتالية لكل حالة من الحالات الأربعة المذكورة أعلاه. وبعدها أيجاد (CPR, PD,and PL) باستخدام الصيغ الرياضية السابقة الذكر وكذلك دراسة البنيات المجهرية لكل حالة من الحالات التآكل الأربعة.

\section{ع ـ النتائج والمناقشة}

من نتائج التجارب و معرفة فرق الوزن للعينات، و بعد أنقضاء ستة أشهر نحصل على الجدول (ॅ). ومنه يتبين بأن الأسلاك تكون بفترة صلاحية أطول عند جو مماتل لفم الأنسان حيث يكون معدل نفاذية التآكل وعمق التتقر منخفض مقارنة" بالحالات الثناث الأخرى والسبب يعود الى طبقة الخمودية المثالية المتكونة على سطح السلك عند الجو المماثل

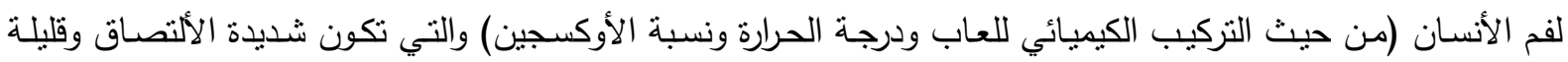

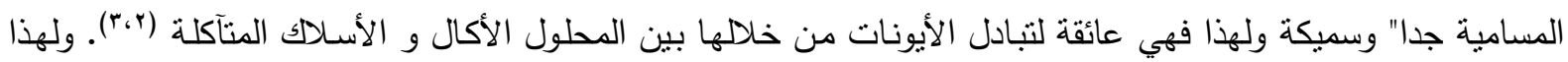

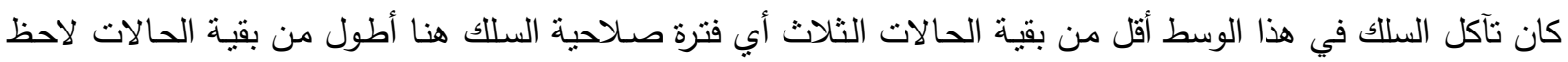

وتليها الأسلاك التي تعمل في اللعاب الصناعي وبنفس التهوية وعند درجة حرارة الغرفة ، فتكون فترة صلاحيتها أقصر من الحالة الأولى والسبب يعود الى إن درجة حرارة الغرفة سوف لن تحفز التفاعل لتكوين طبقة خمودية مثالية تعيق درأف تبادل الأيونات بشكل كافي كما في الحالة السابقة(ه،ه) ـ وبذللك سيكون معدل نفاذية التآكل وعمق التتقر أكبر من الحالة

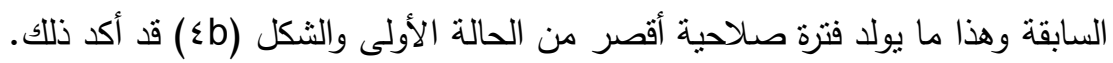

و يعقبها الأسلاك العاملة في محلول كلوريد الصوديوم وبنفس التهوية عند درجة حرارة الغرفة حيث بعطي هذا

الوسط معدل تغلغل للتتقر و عمق تتقر أكبر من الحالتين السابقتين وهذا يعود لدور الأيون الهاليدي أو الهالوجيني (لتهن

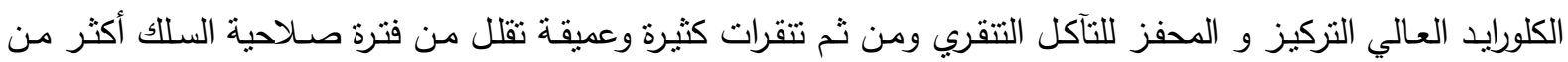

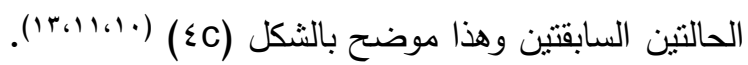
و أقل فترة خدمة تكون للأسلاك العاملة في محلول كلوريد الصوديوم وعند درجة حرارة جسم الأنسان عند توفر

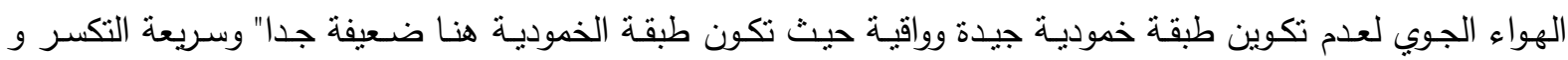

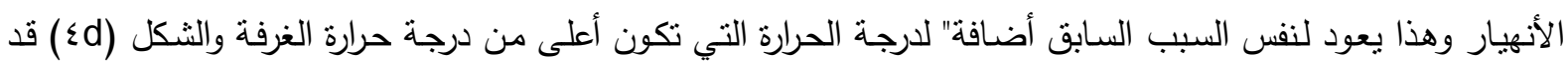

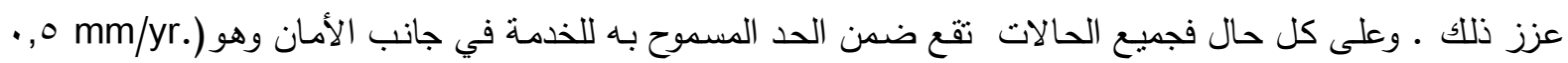

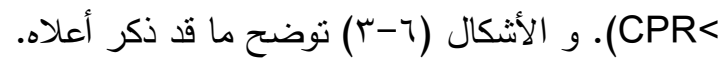

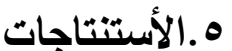

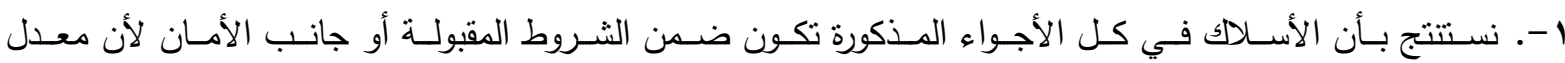

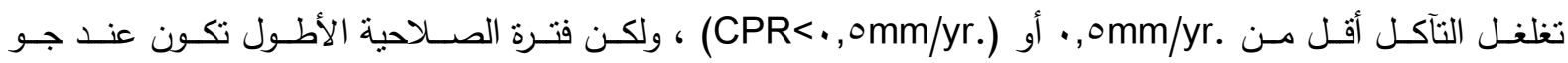

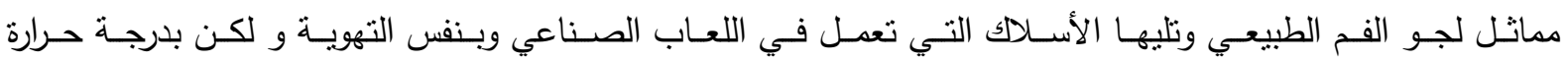

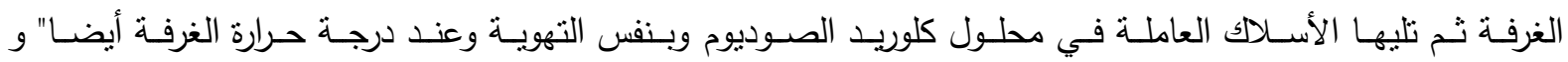




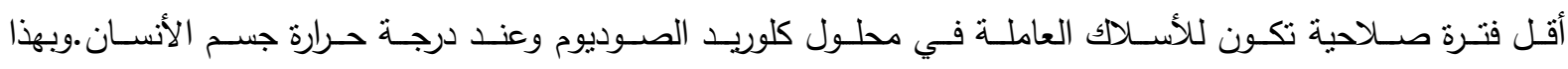

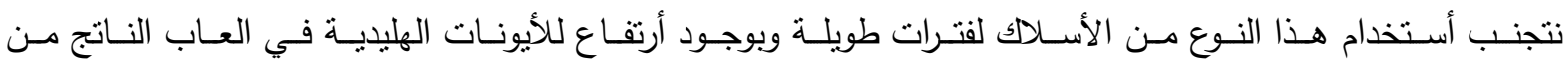

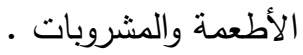

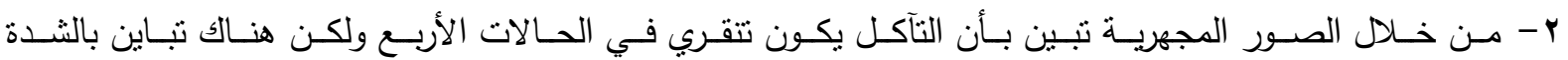

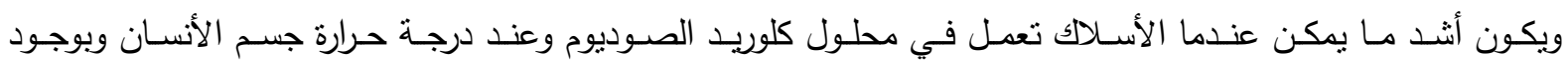

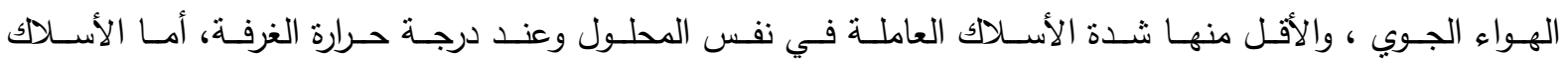

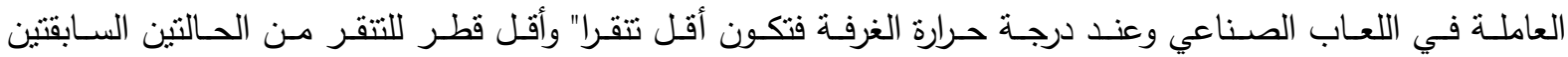

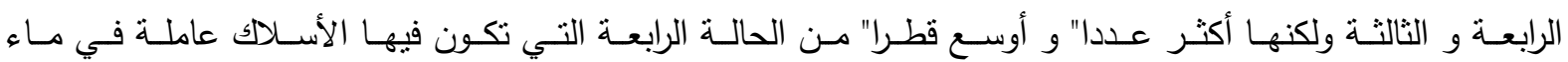

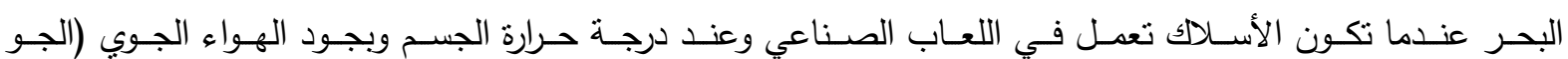
الممانل لفم الأنسان).

المصادر

1. Geetha Manivasagam, Durgalakshmi Dhinasekaran and Asokamani Rajamanickam, "Biomedical Implants: Corrosion and its Prevention - A Review, Recent Patents on Corrosion Science", School of Mechanical and Building Sciences, VIT University, Vellore $7 r$. $1 \leq$, Tamil Nadu, India, $, \cdot 1 \cdot, r, \leq \cdot-0 \leqslant$.

r. J. E. G González, J.C Mirza-Rosca, " Study of the corrosion behavior of titanium and some of its alloys for biomedical and dental implant applications", Original Research Article, Journal of Electro analytical Chemistry, Volume $\{\checkmark 1$, Issue r, Elsevier, ir August 1999, Pages 1.9-110.

r. C. Alves Jr., C.L.B. Guerra Neto, G.H.S. Morais, C.F. da Silva, V. Hajek, " Nitriding of titanium disks and industrial dental implants using hollow cathode Original discharge", Research Article, Surface and Coatings Technology, Volume 19 \&, Issues r-r, Elsevier, 1 May $r . .0$, Pages $197-r \cdot r$.

£. Carlos Nelson Elias, Yoshiki Oshida, José Henrique Cavalcanti Lima, Carlos Alberto Muller, "Relationship between surface properties (roughness, wettability and morphology) of titanium and dental implant removal torque, Original Research Article, Journal of the Mechanical Behavior of Biomedical Materials, Volume ', Issue r, July $r \cdot \lambda$, Pages $r$ r $\leqslant-r \leqslant r$.

•. M.H Fathi, M Salehi, A Saatchi, V Mortazavi, S.B Moosavi, In vitro corrosion behavior of bio ceramic, metallic, and bio ceramic-metallic coated stainless steel dental implants, Original Research Article, Dental Materials, Volume 19, Issue r, May r..r, Pages $1 \wedge \Lambda_{-}$ 191 . 
7. Z. Schwartz, A.L. Raines, B.D. Boyan,"The Effect of Substrate Micro topography on Osseo integration of Titanium Implants", Comprehensive Biomaterials, Volume $`, ~ \uparrow .11$, Pages ris-ror.

v. P. Layrolle, "Calcium Phosphate Coatings", Comprehensive Biomaterials, Volume ', r. Il, Pages rrr-rrq.

^. L. Reclaru, J.-M. Meyer, "Study of corrosion between a titanium implant and dental alloys", Journal of Dentistry, Volume $r$, Issue $r$, June 199 \&, Pages $109 \_171$.

१. Fontana and Green, "Corrosion Engineering", McGraw-Hill book Co., third edition, $(1911)$

1. Shrier L. L., "Corrosion Metal / Environment reactions", Volume ।, printed and bound in Great Britain, Butterworth Hejne Mann, Third edition, ( 99 १ ), Reprinted ( $\uparrow \cdots$ ).

11. Kenneth R. \& John chamberlain, "Corrosion for Science \& Engineering", Addison Wesley London, LONGMAN Group Limited, first and second edition, (19^^_1990), Reprinted (1997).

Ir. Denny A. Jones. "Principles and Prevention of Corrosion", Macmillan. Publishing Co. Maxwell Macmillan Canada \& Maxwell international Publishing Group, ( 994 ). 1r. Annual Book of ASTM Standards, Vol. $\cdot r, \cdot r$, Designation Grl, $(r \cdot 1 \cdot)$.

جدول (1): بيين التركيب الكيميائي للفولاذ الأوستتايتي المستخدم كأسلاك لنقويم الأسنان .

\begin{tabular}{|c|c|c|c|c|c|c|c|c|}
\hline $\mathrm{Fe}$ & $\mathrm{Si}$ & W & V & $\mathrm{Mn}$ & $\mathrm{Ni}$ & $\mathrm{Cr}$ & C & Elements العناصر \\
\hline Rem. & $\cdot, .0$ & $\cdot, \cdots r$ & $\cdot, \ldots q$ & $1, v_{0}$ & $7, \varepsilon \varepsilon$ & $17, \mathrm{r} \wedge$ & ס ס ו, & \%Wt نسبته المئوية \\
\hline & & & & & & & & \\
\hline
\end{tabular}

جدول (ץ): التركيب الكيميائي للعاب الصناعي (Artificial Saliva).

\begin{tabular}{|c|c|c|}
\hline النسبة بالـ (gr./L) & المادة & ت \\
\hline$\cdot, \varepsilon$ & $\mathrm{NaCl}$ & 1 \\
\hline$\cdot, \varepsilon$ & $\mathrm{KCl}$ & $r$ \\
\hline$\cdot, \vee \vee 90$ & $\mathrm{CaCl}_{r} .{ }^{\mathrm{r}} \mathrm{H}_{r} \mathrm{O}$ & $r$ \\
\hline
\end{tabular}


دراسة تحليد أطول فترة صلاحية لأسلاك تقويم الأسنان في أجواء مختلفة

\begin{tabular}{|c|c|c|}
\hline$\cdot, 79$ & $\mathrm{NaH}_{\curlyvee} \mathrm{PO} \varepsilon$ & \\
\hline$\cdot, 19$ & $\mathrm{MgCl} r$ & 0 \\
\hline $1, \cdots$ & Urea & 7 \\
\hline l... ml. & Distilled Water & $\mathrm{v}$ \\
\hline
\end{tabular}

جدول (؟): المقارنة بين حالات التآكل الأربعة من حيث معدل نفاذ التآكل وعمق التتقر وفترة صلاحية السلك.

\begin{tabular}{|c|c|c|c|}
\hline PL (yr.) & PD $(\mu \mathrm{m})$. & CPR $(\mu \mathrm{m} / \mathrm{yr})$. & الحالة \\
\hline$P L I=70, r Y \Sigma$ & PDI $=\cdot, V M r$ & CPR $1=1, \leq 7 \leq$ & جو مماتل لفم الأنسان \\
\hline$P L r=r r, q \wedge r$ & $P D Y=1, \leqslant 7 \leqslant$ & $C P R r=r, q r \wedge$ & اللعاب الصناعي وعند \\
\hline$P L Y=10, \wedge T r$ & $P D r=r, q r \wedge$ & CPRY $=0, \wedge 01$ & في محلول كلوريد الصوديوم \\
\hline$P L \varepsilon=1,, \vee \vee \vee \wedge$ & $P D \varepsilon=\varepsilon, \Gamma q \uparrow$ & $\mathrm{CPR} \varepsilon=\wedge, \vee \wedge \varepsilon$ & في محلول كلوريد الصوديوم \\
\hline
\end{tabular}

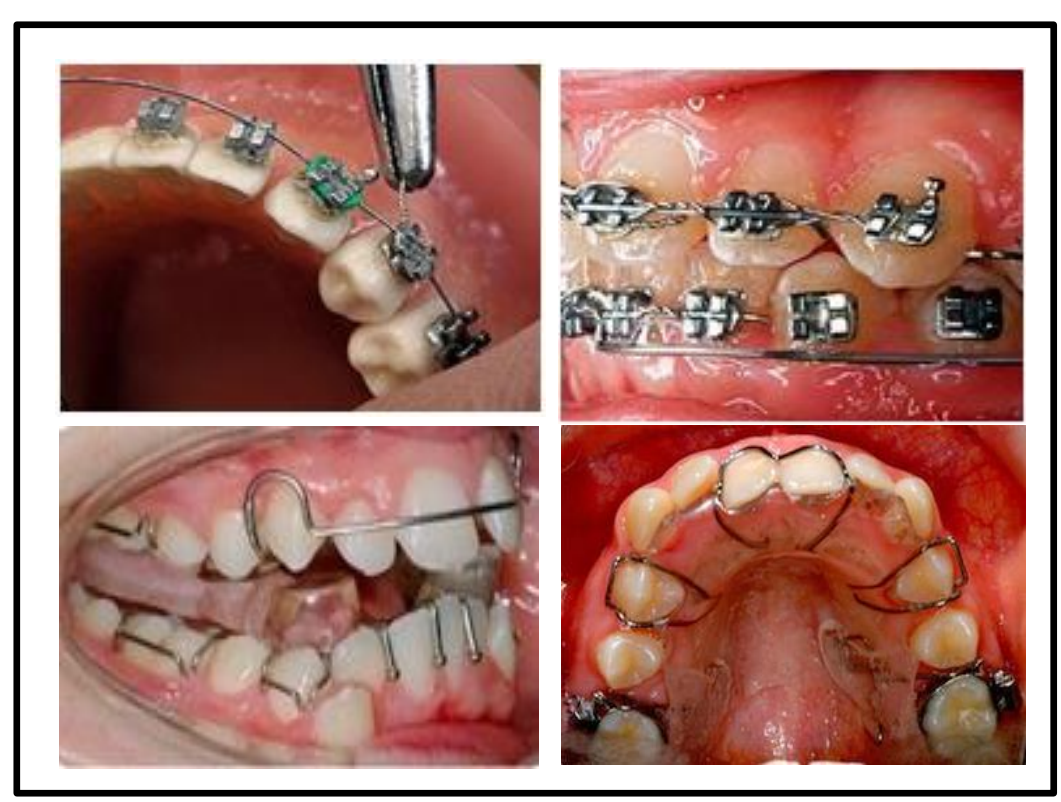

الثكل (1): أسلاك تقويم الأسنان في أماكن مختلفة من الفم (־). 


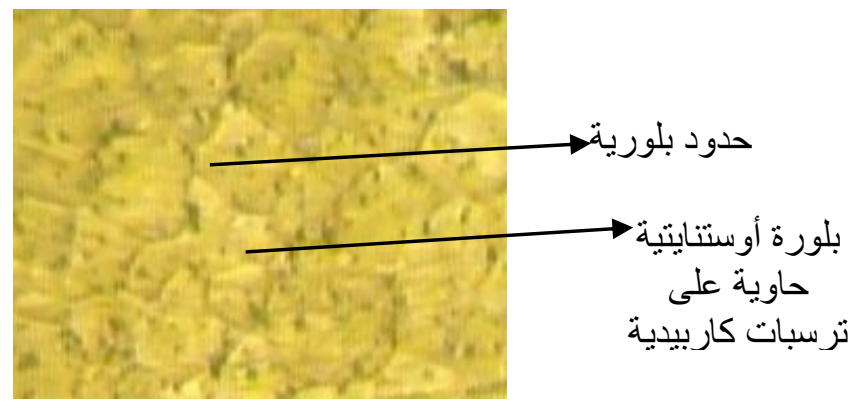

الشكل (ץ): البنية المجرية لأسلاك تقويم الأسنان المستخدمة ومن نوع ( • ب) فولاذ أوستتايتي مقاوم للصدأ وبقوة تكبير .$(0 . . X)$

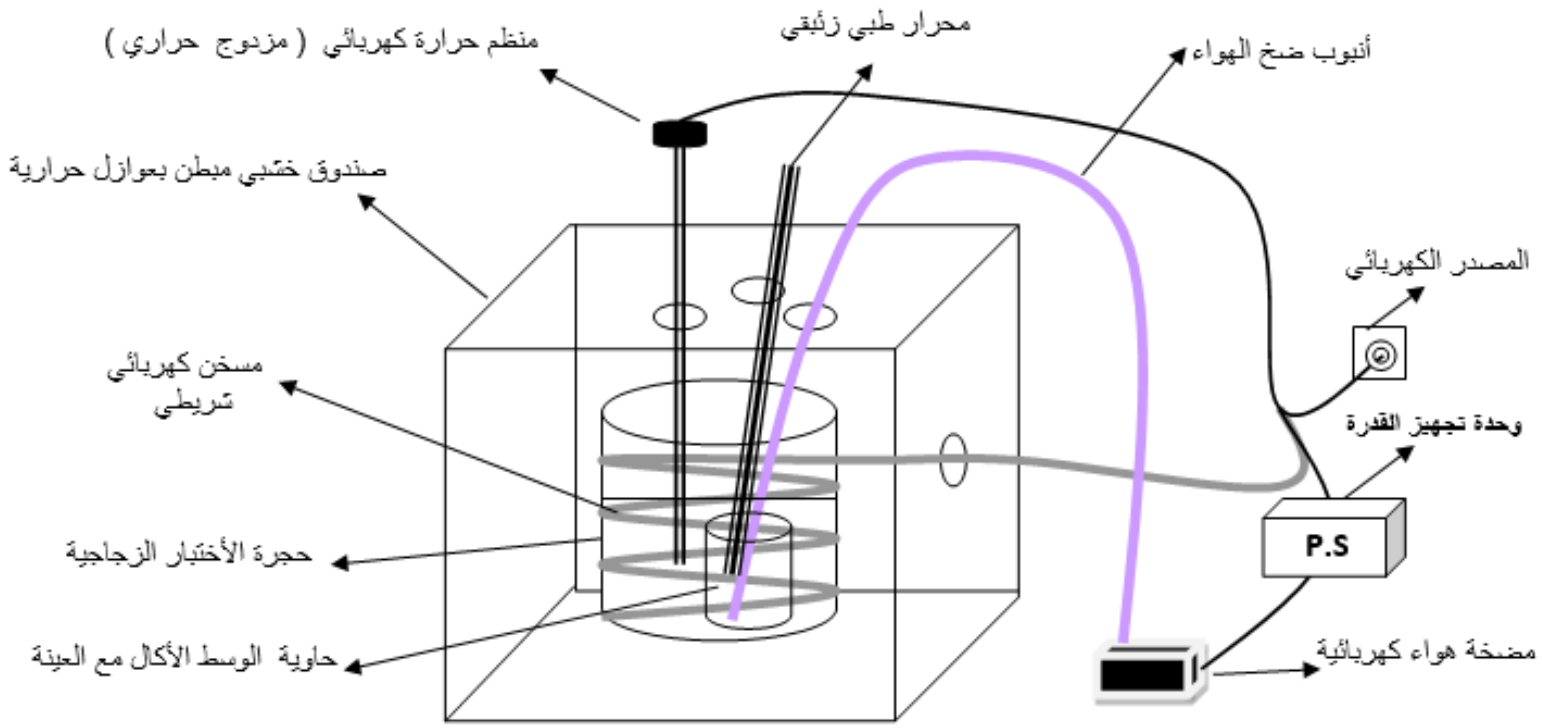

شكل (ra): مخطط لمنظومة الأختبار المستعملة (المصممة والمصنعة محليا").

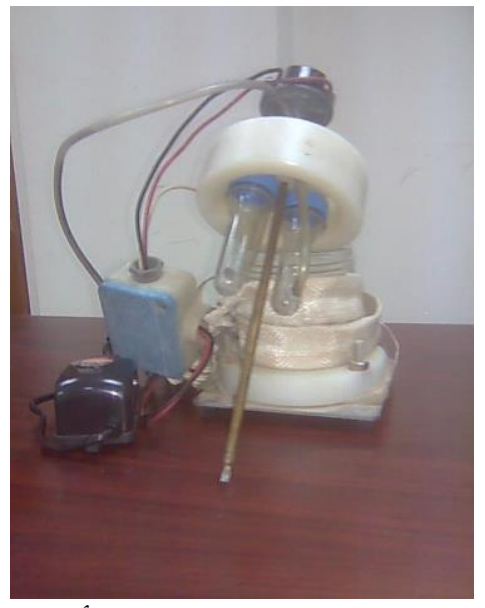

شكل (rC): صورة فوتغرافية لمنظومة أختبار مفتوحة.

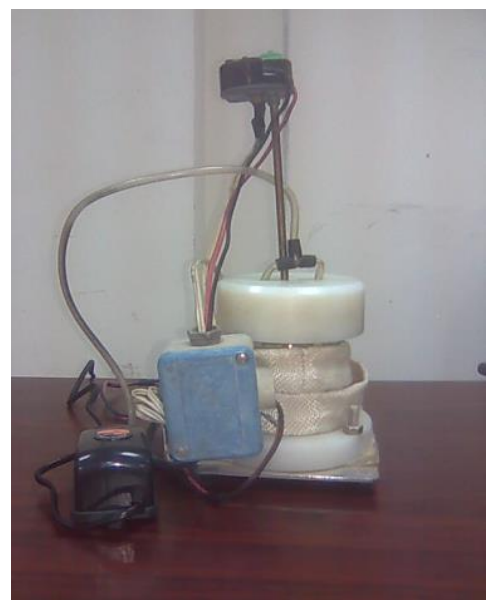

شكل (rb) صورة فوتغرافية لمنظومة أختبار منصوبة.

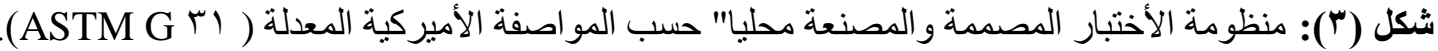




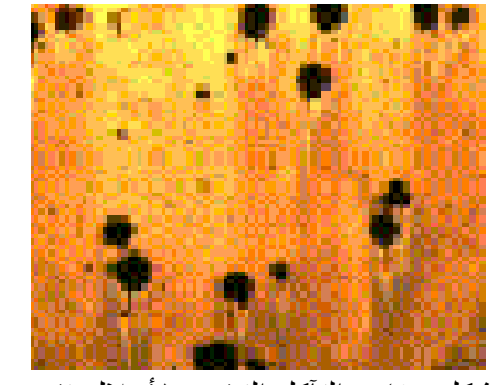

شكل (b؛ ): التآكل التنقري لأسلاك تقويم

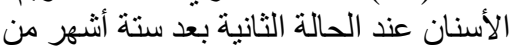
الأختبار وبقوة تكبير (X) (OX).

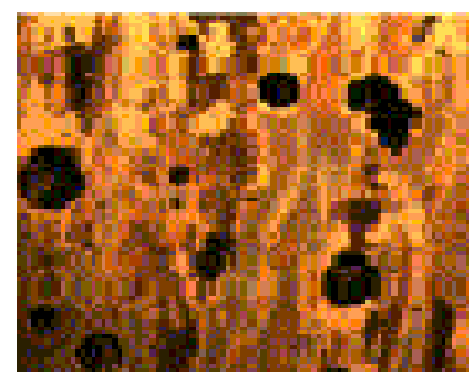

شكل (dई ) التآكل التنقري لأسلاك تقويم الأسنان عند الحالة الر ابعة بعد ستخة أثشهر من الأختبار

$$
\text { وبقوة تكبير (X) (OX). }
$$

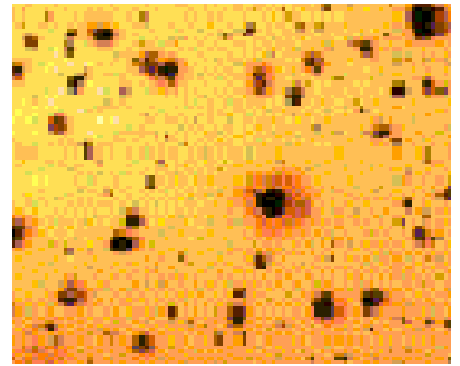

شكل (a ) : التآكل التتقري لأسلاك تقويم الأسنان عند الحالة الأولى بعد ستة أنشهر من الأختبار

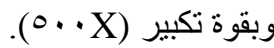

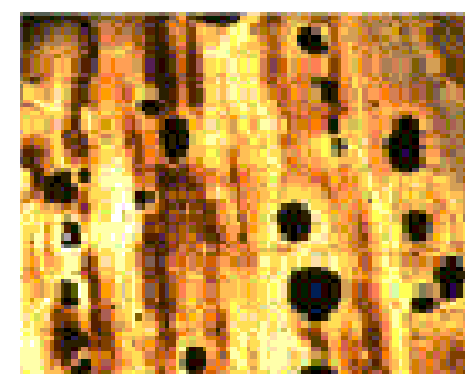

شكل (c) ): التآكل التتقري لأسلاك تقويم الأسنان عند الحالة الثالثة بعد ستُة أثنهر من الأختبار وبقوة تكبير (X) (O.X).

شكل (؛) ): صور مجهرية لأسلاك تقويم الأسنان بعد ستة أثهر من حالات التآكل الأربع.

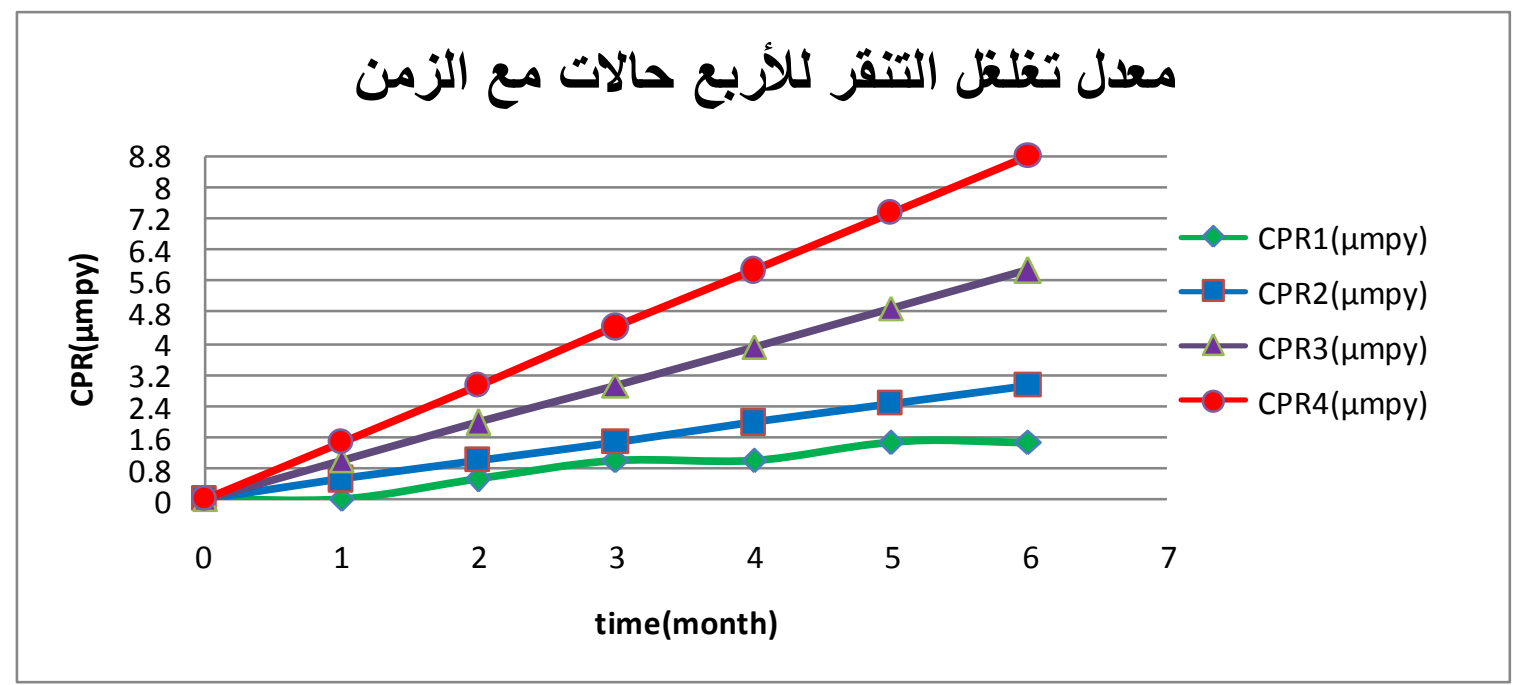

شكل (•): معدل تغلغل التآكل التتقري في الأربع حالات بعد مرور ستة أثنر. 

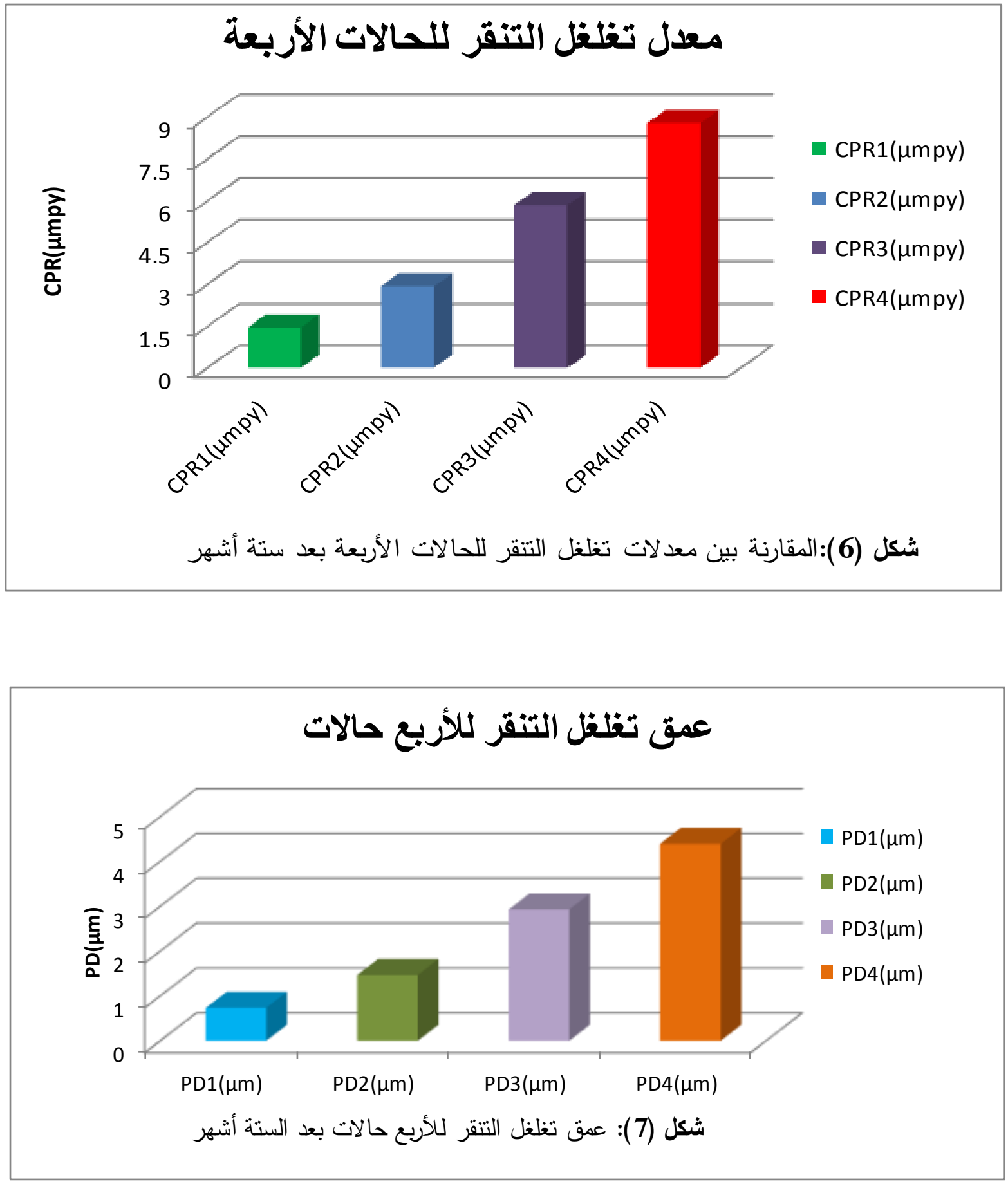


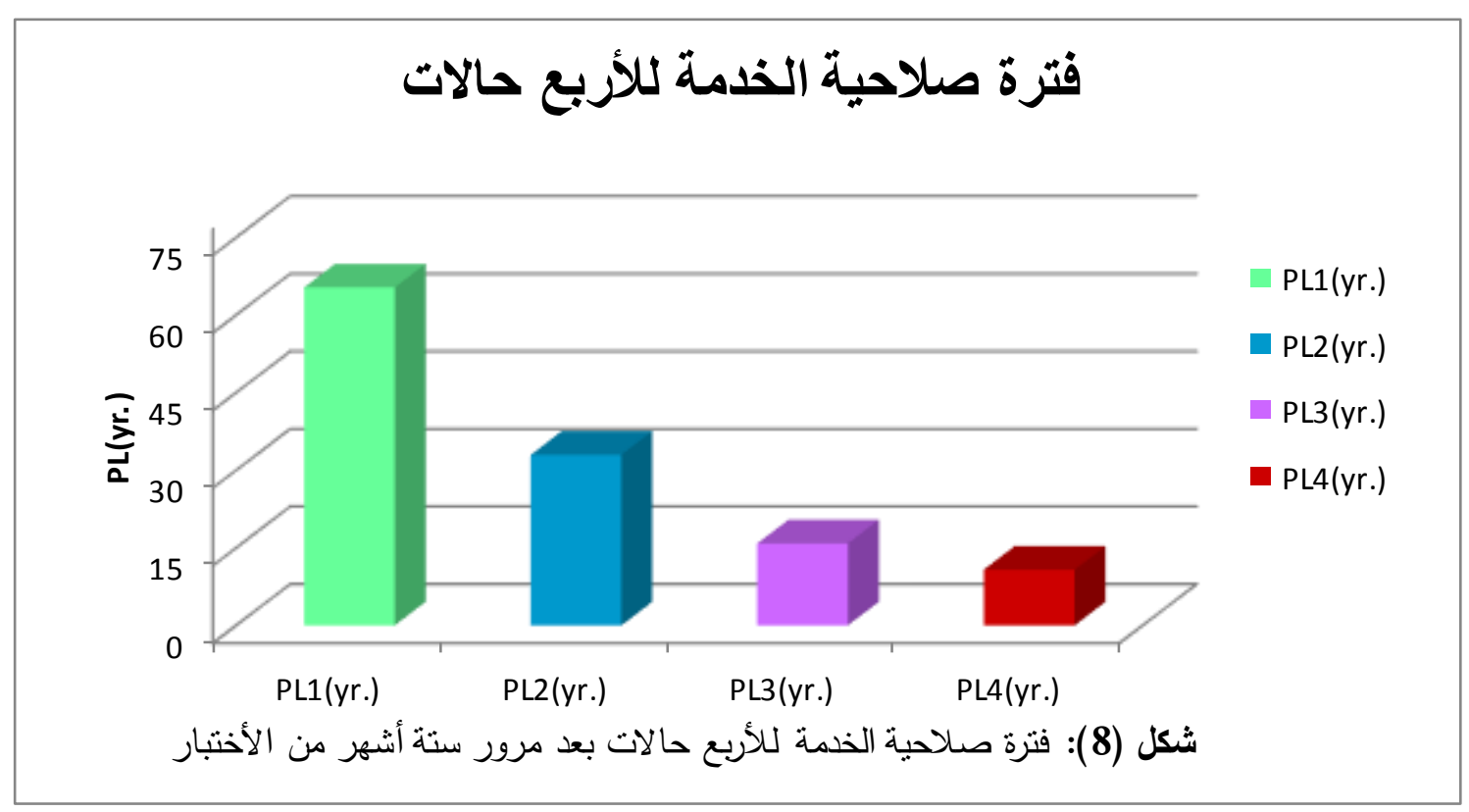


دراسة تحديد أطول فترة صلاحية لأسلاك تقويم الأسنان في أجواء مختلفة

\title{
STUDY TO DETERMINE THE LONGEST VALIDITY PERIOD FOR ORTHODONTIC WIRES IN A DIFFERENT ENVIRONMENTS
}

\author{
Mohammed Abdulateef Ahmed \\ Production and Metallurgy Engineering Department \\ University of Technology \\ Metal_metalı^@yahoo.com
}

\begin{abstract}
:
The research summary to knowing longest period of validity for orthodontic wires in four corrosive media, the first medium approximately similar to human mouth such as corrosive medium (Artificial Saliva), human body temperature, hydrogen power $(\mathrm{pH})$, and aeration , and compared with orthodontic wires these serve in same corrosive medium but at room temperature and the identical aeration as a second corrosive medium, and compared to orthodontic wires present or attending in sodium chloride solution instead of artificial saliva at room temperature and the like aeration as a third corrosive medium. The final comparison test with orthodontic wires present in sodium chloride solution at human body temperature and the like aeration too.

The study done by using device or system was native designed and manufactured according to modified American standard specification (ASTM G ${ }^{\mu}$ ), the orthodontic wires were from type austenitic stainless steel $(r \cdot 1)$.

After four mentioned corrosion experiments for serial six months period , and usage Corrosion Penetration Rate law (CPR) and by traditional mathematical methods addition to the microstructures of samples before and after corrosion experiments, conclude that ; the longest validity period for orthodontic wires at environment approximately similar to human mouth ,then wires worked in artificial saliva at room temperature, followed by the wires served in sodium chloride solution at room temperature, finally; orthodontic wires were worked in sodium chloride solution at human body temperature had shortest validity period.
\end{abstract}

Key words: Orthodontic, Biomaterials, stainless steel, Austenitic steel, Artificial saliva. 